\title{
In Vitro Activity of OPT-80 against Clostridium difficile
}

\author{
Grit Ackermann,* Birgit Löffler, Daniela Adler, and Arne C. Rodloff \\ Institute for Medical Microbiology and Epidemiology of Infectious Diseases, University of Leipzig, \\ 04103 Leipzig, Germany
}

Received 9 December 2003/Returned for modification 26 January 2004/Accepted 11 February 2004

\begin{abstract}
Clostridium difficile remains the major cause of nosocomial diarrhea. Reports on impaired susceptibility of $C$. difficile to metronidazole and vancomycin and frequent relapses of patients after therapy necessitate the search for new substances. With this study, the activity of OPT-80, a new macrocycle, against 207 C. difficile strains and against other obligately anaerobic bacteria was tested. OPT-80 showed high in vitro activity against all $C$. difficile strains tested.
\end{abstract}

Clostridium difficile is the major cause of nosocomial diarrhea. Patients suffering from $C$. difficile-associated diarrhea (CDAD) are burdened with a prolonged hospital stay, and an increase in costs has been reported for these patients due to additional diagnostic and therapeutic procedures (5). First-line antimicrobials for the treatment of CDAD are metronidazole and vancomycin. Meanwhile, diminished susceptibility was observed for both substances $(1,4,8,12)$; however, this was not directly linked to treatment failures. On the other hand, as many as $20 \%$ of patients had at least one recurrence of CDAD after the initial therapy was discontinued (3). Another study reported recurrence rates of 41 to $50 \%$ that were not significantly different for patients treated with vancomycin or metronidazole (6). Currently, strategies for reconstituting and balancing of the gut flora to increase colonization resistance as well as identification of new antibacterial compounds are under study.

OPT-80 (Optimer Pharmaceuticals, Inc., San Diego, Calif.) is a novel macrocycle. OPT-80 has an unsaturated 18 -member core bearing a seven-carbon sugar at carbon 11 and a 6-deoxy sugar at carbon 20. Also, OPT-80 has been shown to inhibit RNA synthesis by the Escherichia coli and Bacillus subtilis RNA polymerases (9). OPT-80 shows little or no systemic absorption (10). Earlier studies showed some activity against gram-positive aerobic and anaerobic organisms. Gram-negative species were not inhibited by OPT-80 (11). Swanson et al. (10) studied the activity of OPT-80 in vitro against 15 strains of C. difficile and observed MICs with a range of 0.12 to 0.25 $\mu \mathrm{g} / \mathrm{ml}$. In a hamster model for pseudomembranous colitis, OPT-80 was capable of completely preventing the lethality of the animals and prevented the occurrence of relapses (10).

With the present study, we assessed the in vitro activity of OPT-80 against clinical isolates (collected from 1986 to 2002) to $C$. difficile $(n=207)$, Bacteroides fragilis $(n=69)$, Prevotella spp. $(n=35)$, Eubacterium spp. $(n=26)$, Propionibacterium acnes $(n=16)$, and Lactobacillus spp. $(n=8)$. C. difficile strains were isolated from patients of the Leipzig University

\footnotetext{
* Corresponding author. Mailing address: Institute for Medical Microbiology and Epidemiology of Infectious Diseases, University of Leipzig, Liebigstrasse 24, 04103 Leipzig, Germany. Phone: 49341 9715200. Fax: 49341 9715209. E-mail: ackermg@medizin.uni-leipzig .de.
}

Hospital, a community hospital in Leipzig, and the Bonn University Hospital. The prevotellae, bacteroides, eubacteria, lactobacilli, and propionibacteria were isolated either at the R. M. Alden Research Laboratory in Santa Monica, Calif., or at our institute. The bacterial strains were identified with the RapID ANA II system, the PRO KIT (both from REMEL Inc., Norcross, Ga.) or by the use of prereduced anaerobically sterilized biochemicals (Anaerobe Systems, Morgan Hill, Calif.). Reference strains used in this study were C. difficile VPI 10463, B. fragilis ATCC 25285, Eubacterium lentum ATCC 43055, and Staphylococcus aureus ATCC 29213.

Antimicrobial agents were obtained from the manufacturers as laboratory powders of known potency as follows: OPT-80 was from Optimer Pharmaceuticals, Inc., vancomycin and metronidazole were from Sigma Chemical (Taufkirchen, Germany), moxifloxacin was from Bayer (Wuppertal, Germany), linezolid was from Pharmacia Biotech (Cambridge, United Kingdom), and fusidic acid was from Boehringer (Ingelheim, Germany).

Antimicrobial susceptibility testing was performed using either broth microdilution and/or agar dilution validated according to the recommendations of the Deutsches Institut fuer Normung and the National Committee for Clinical Laboratory Standards (NCCLS), respectively $(2,7)$. Broth microdilution was used for all $C$. difficile strains and vancomycin, metronidazole, moxifloxacin, fusidic acid, linezolid, and OPT-80. In order to confirm the results received with the broth microdilution technique, agar dilution was performed for 25 of the tested $C$. difficile strains and OPT-80. All other anaerobes included in this study were tested with OPT-80 and the agar dilution method.

MICs were determined by the broth microdilution technique with 96-well microdilution plates. The plates were filled with $100 \mu \mathrm{l}$ of Wilkins-Chalgren broth (Oxoid; Unipath Ltd., Basingstoke, United Kingdom) per well containing the final antibiotic concentrations. The plates were stored at $-80^{\circ} \mathrm{C}$ until use. Plates were thawed and preincubated for $3 \mathrm{~h}$ in an anaerobic chamber (WA 6200; Heraeus Instruments, Hanau, Germany) containing an atmosphere of $80 \% \mathrm{~N}_{2}, 15 \% \mathrm{CO}_{2}$, and $5 \% \mathrm{H}_{2}$. The bacterial inocula were prepared by suspending growth from 48 -h cultures in Wilkins-Chalgren broth. The bacteria were delivered by a semiautomatic inoculator (MIC-2000; Dynatech Laboratories, Inc., Chantilly, Va.). The final inocu- 
TABLE 1. Distribution of MIC values for 207 C. difficile strains

\begin{tabular}{|c|c|c|c|c|c|c|c|c|c|c|c|c|c|c|c|c|}
\hline \multirow{2}{*}{ Agent } & \multicolumn{16}{|c|}{ No. of strains with MIC $(\mu \mathrm{g} / \mathrm{ml})$ of: } \\
\hline & $\leq 0.0009$ & 0.0019 & 0.0039 & 0.0078 & 0.0156 & 0.0312 & 0.0625 & 0.125 & 0.25 & 0.5 & 1 & 2 & 4 & 8 & 16 & $\geq 32$ \\
\hline Vancomycin & & & & & 1 & & 3 & 7 & 81 & 115 & & & & & & \\
\hline Metronidazole & & & 2 & 2 & 5 & 16 & 39 & 138 & 3 & 2 & & & & & & \\
\hline Moxifloxacin & & & & & & & 1 & 1 & 2 & 10 & 56 & 109 & 2 & 7 & 12 & 7 \\
\hline Fusidic acid & & & & & 65 & 87 & 44 & 9 & 1 & & & 1 & & & & \\
\hline Linezolid & & & & & & & 7 & 14 & 35 & 50 & 87 & 10 & 4 & & & \\
\hline OPT-80 & 75 & 42 & 49 & 21 & 16 & 1 & 3 & & & & & & & & & \\
\hline
\end{tabular}

lum was approximately $1.0 \times 10^{5} \mathrm{CFU} /$ well. The plates were incubated for $48 \mathrm{~h}$ at $37^{\circ} \mathrm{C}$ in the anaerobic chamber. The MIC was defined as the lowest antibiotic concentration that inhibited visible growth.

Brucella agar supplemented with $5 \mathrm{mg}$ of hemin/liter, $1 \mathrm{mg}$ of vitamin $\mathrm{K} /$ liter, and $5 \%$ laked horse blood (Oxoid) was used for the agar dilution technique. Serial doubling dilutions $(0.0009$ to $128 \mu \mathrm{g} / \mathrm{ml})$ were prepared for all compounds tested. Dilutions of the antibiotics were made according to standard procedures on the day of the test. Inocula were prepared according to NCCLS standards, with a final inoculum density of approximately $10^{5} \mathrm{CFU} /$ spot (7). A semiautomatic replicator device (A400 Multipoint Inoculator; Bachhofer $\mathrm{GmbH}$, Reutlingen, Germany) was used to apply the individual inocula. Plates were incubated at $37^{\circ} \mathrm{C}$ in an anaerobic atmosphere for $48 \mathrm{~h}$, and MICs were defined as described above.

OPT-80 was found to be very soluble in dimethyl sulfoxide (DMSO), moderately soluble in ethanol, and insoluble in water. For 36 strains (11 Eubacterium spp. and $25 \mathrm{C}$. difficile isolates), agar dilution was run in parallel with (i) OPT-80 dissolved and diluted in DMSO, (ii) OPT-80 dissolved in DMSO and diluted in water, and (iii) OPT-80 dissolved in ethanol and diluted in water.

According to NCCLS recommendations, resistance was defined as follows: metronidazole MIC, $\geq 32 \mathrm{mg} /$ liter (7). Since the NCCLS has not yet approved breakpoints for vancomycin, moxifloxacin, linezolid, and fusidic acid and anaerobes, in this study the following MICs were interpreted as resistance: vancomycin, $\geq 16 \mu \mathrm{g} / \mathrm{ml}$; moxifloxacin, $\geq 8 \mu \mathrm{g} / \mathrm{ml}$; linezolid, $\geq 4$ $\mu \mathrm{g} / \mathrm{ml}$; fusidic acid, $\geq 4 \mu \mathrm{g} / \mathrm{ml}$.

OPT-80 showed high activity against all $C$. difficile strains tested (MIC $\leq 0.0625 \mu \mathrm{g} / \mathrm{ml}$ ). The very low MICs were confirmed testing 26 strains with the agar dilution technique. MICs were much lower when OPT-80 was (i) dissolved and diluted in DMSO, compared to the results received with OPT-80 dissolved in either (ii) DMSO or (iii) ethanol and diluted in water. The latter ones were similar to those established by broth microdilution and confirmed the very low MICs for susceptible strains.

All $C$. difficile strains were susceptible to vancomycin, metronidazole, and fusidic acid (Tables 1 and 2). A total of $13 \%$ ( $n$ $=26)$ of the strains showed resistance to moxifloxacin, and four strains $(2 \%)$ exhibited elevated MICs to linezolid (MIC = $4 \mathrm{mg} /$ liter). Eubacteria and gram-negative anaerobic bacteria were not susceptible to OPT-80 (Table 3), exhibiting values for the MIC at which $50 \%$ of isolates were inhibited $\left(\mathrm{MIC}_{50}\right)$ and the $\mathrm{MIC}_{90}$ of 32 and $\geq 128 \mathrm{mg} /$ liter and of $\geq 128$ and $\geq 128$ $\mathrm{mg} /$ liter, respectively. The numbers of lactobacilli and propionibacteria tested were too small to make firm conclusions on the activity of OPT-80 against these species.

For the reference strains, the MICs of metronidazole, vancomycin, moxifloxacin, and linezolid were all within the acceptable ranges described by the NCCLS (7).

The MICs of OPT- 80 for $C$. difficile reported by Theriault et al. and Swanson et al. were higher than those determined in this study $(10,11)$. The treatment of CDAD with OPT-80 in a hamster model published by Swanson et al. showed a higher efficacy than the treatment with vancomycin. No OPT-80 was detected in the sera of the animals after oral treatment with 25 $\mathrm{mg} / \mathrm{kg}$ of body weight. Antibiotic levels in the ceca of the hamsters were reported to be $248 \mu \mathrm{g} / \mathrm{g}$ at a 6 -h time point. Doses as low as $0.2 \mathrm{mg} / \mathrm{kg}$ prevented the development of fatal colitis in clindamycin-treated hamsters (10). Since OPT-80 lacks activity against gram-negative bacteria, its potency to affect the colonization resistance within the gut must be anticipated to be low.

TABLE 2. Cumulative percentage of strains inhibited at a given MIC $(n=207)$

\begin{tabular}{|c|c|c|c|c|c|c|c|c|c|c|c|c|c|c|c|c|}
\hline \multirow{2}{*}{ Antimicrobial } & \multicolumn{16}{|c|}{ Cumulative $\%$ strains inhibited at MIC $(\mu \mathrm{g} / \mathrm{ml})$ of: } \\
\hline & $\leq 0.0009$ & 0.0019 & 0.0039 & 0.0078 & 0.0156 & 0.0312 & 0.0625 & 0.125 & 0.25 & 0.5 & 1 & 2 & 4 & 8 & 16 & $\geq 32$ \\
\hline Vancomycin & & & & & 1 & 1 & 2 & 5 & 45 & 100 & & & & & & \\
\hline Metronidazole & & & 1 & 2 & 4 & 12 & 31 & 98 & 99 & 100 & & & & & & \\
\hline Moxifloxacin & & & & & & & 1 & 1 & 2 & 7 & 34 & 86 & 87 & 91 & 97 & 100 \\
\hline Fusidic acid & & & & & 32 & 73 & 95 & 99 & 99 & 99 & 99 & 100 & & & & \\
\hline Linezolid & & & & & & & 3 & 10 & 27 & 51 & 94 & 98 & 100 & & & \\
\hline OPT-80 & 36 & 57 & 80 & 90 & 98 & 99 & 100 & & & & & & & & & \\
\hline
\end{tabular}


TABLE 3. Distribution of MIC values for OPT-80 and five bacterial species: B. fragilis $(n=69)$, Prevotella spp. $(n=35)$, Eubacterium spp. $(n=26)$, Lactobacillus spp. $(n=8)$, and P. acnes $(n=16)$

\begin{tabular}{|c|c|c|c|c|c|c|c|c|c|c|c|c|c|c|}
\hline \multirow{2}{*}{ Organism } & \multicolumn{13}{|c|}{ No. of isolates with MIC $(\mu \mathrm{g} / \mathrm{ml})$ of: } & \multirow{2}{*}{$\mathrm{MIC}_{50} / \mathrm{MIC}_{90}$} \\
\hline & $\leq 0.0312$ & 0.0625 & 0.125 & 0.25 & 0.5 & 1 & 2 & 4 & 8 & 16 & 32 & 64 & $\geq 128$ & \\
\hline Bacteroides fragilis & & & & & & & & & & & & & 69 & $\geq 128 / \geq 128$ \\
\hline Prevotella spp. & & & & & & & & & & 1 & & 2 & 32 & $\geq 128 / \geq 128$ \\
\hline Eubacterium spp. & 1 & 3 & & & & & & & 1 & 6 & 8 & 3 & 4 & $32 / \geq 128$ \\
\hline Lactobacillus spp. & & & & & & & & & 5 & 2 & 1 & & & $\mathrm{NA}^{a}$ \\
\hline Propionibacterium acnes & 1 & 1 & & & & & 2 & 5 & & 2 & 3 & & 2 & $4 / \geq 128$ \\
\hline
\end{tabular}

${ }^{a}$ NA, not applicable.

OPT-80 is a new compound with promising characteristics for the therapy of CDAD. Clinical studies are needed to confirm the results presented here.

We thank M. Linder, St. Georg Hospital, Leipzig, Germany, and E. J. C. Goldstein, R. M. Alden Research Laboratory, Los Angeles, Calif., for supplying bacterial strains for this study. The compound was supplied by the manufacturer (Optimer Pharmaceuticals, Inc.).

\section{REFERENCES}

1. Brazier, J. S., W. Fawley, J. Freeman, and M. H. Wilcox. 2001. Reduced susceptibility of Clostridium difficile to metronidazole. J. Antimicrob. Chemother. 48:741-742.

2. Deutsches Institut für Normung. 2000. Medizinische Mikrobiologie und Immunologie: Diagnostische Verfahren. Deutsches Institut für Normung, Berlin, Germany.

3. Fekety, R., L. V. McFarland, C. M. Surawicz, R. N. Greenberg, G. W. Elmer, and M. E. Mulligan. 1997. Recurrent Clostridium difficile diarrhea: characteristics of and risk factors for patients enrolled in a prospective, randomized, double-blinded trial. Clin. Infect. Dis. 24:324-333.

4. Jang, S. S., L. M. Hansen, J. E. Breher, D. A. Riley, K. D. Madigan, Y. J. Tang, J. Silva, Jr., and D. C. Hirsh. 1997. Antimicrobial susceptibilities of equine isolates of Clostridium difficile and molecular characterization of metronidazole-resistant strains. Clin. Infect. Dis. 25:S266-S267.

5. Kyne, L., M. B. Hamel, R. Polavaram, and C. P. Kelly. 2002. Health care costs and mortality associated with nosocomial diarrhea due to Clostridium difficile. Clin. Infect. Dis. 34:346-353.

6. McFarland, L. V., C. M. Surawicz, M. Rubin, R. Fekety, G. W. Elmer, and R. N. Greenberg. 1999. Recurrent Clostridium difficile disease: epidemiology and clinical characteristics. Infect. Control Hosp. Epidemiol. 20:43-50.

7. National Committee for Clinical Laboratory Standards. 1997. Methods for antimicrobial susceptibility testing of anaerobic bacteria, 4th ed. Approved standard M11-A4. National Committee for Clinical Laboratory Standards, Wayne, PA

8. Peláez, T., L. Alcala, R. Alonso, M. Rodriguez-Creixems, J. M. GarciaLechuz, and E. Bouza. 2002. Reassessment of Clostridium difficile susceptibility to metronidazole and vancomycin. Antimicrob. Agents Chemother. 46:1647-1650.

9. Sergio, S., G. Pirali, R. White, and F. Parenti. 1975. Lipiarmycin, a new antibiotic from actinoplanes. III. Mechanism of action. J. Antibiot. 28:543549.

10. Swanson, R. N., D. J. Hardy, N. L. Shipkowitz, C. W. Hanson, N. C. Ramer, P. B. Fernandes, and J. J. Clement. 1991. In vitro and in vivo evaluation of tiacumicins B and C against Clostridium difficile. Antimicrob. Agents Chemother. 35:1108-1111.

11. Theriault, R. J., J. P. Karwowski, M. Jackson, R. L. Girolami, G. N. Sunga, C. M. Vojtko, and L. J. Coen. 1987. Tiacumicins, a novel complex of 18membered macrolide antibiotics. J. Antibiot. 40:567-574.

12. Wong, S. S.-S., P. C.-Y. Woo, W. K. Luk, and K.-Y. Yuen. 1999. Susceptibility testing of Clostridium difficile against metronidazole and vancomycin by disk diffusion and Etest. Diagn. Microbiol. Infect. Dis. 34:1-6. 